\title{
Le Vide, pays de frontière de Roland Barthes
}

\author{
Willy Paillé \\ Université Bordeaux Montaigne
}

Ici commence un voyage en sept stations à
travers: un pays, une histoire, une
expérience, un concept, une sensibilité, un
avenir - peut-être une crainte, une
nostalgie.

Barthes, 2002, t. 5, p. 479.

Pays de frontière se dit d'une place forte qui fait front à l'ennemi. Un tel pays, dans l'œuvre de Barthes, pourrait correspondre au Japon, L'Empire des signes lui étant pleinement consacré. Cela dit, Barthes défend ici moins un pays que l'arrière-pays, son paysage (au sens phénoménal et nouménal du terme) : le Vide, contre le Plein magnifié, mythifié par l'origine, l'identité et la langue même de l'auteur (qui sont aussi les nôtres) : 
En n'importe quel endroit de ce pays, il se produit une organisation spéciale de l'espace : voyageant (dans la rue, en train le long des banlieues, des montagnes), j'y perçois la conjonction d'un lointain et d'un morcellement, la juxtaposition de champs (au sens rural et visuel) à la fois discontinus et ouverts (des parcelles de théiers, de pins, de fleurs mauves, une composition de toits noirs, un quadrillage de ruelles, un agencement dissymétriques de maisons basses) : nulle clôture (sinon très basse) et cependant je ne suis jamais assiégé par l'horizon (et son relent de rêve) : aucune envie de gonfler les poumons, de bomber la poitrine pour assurer mon moi, pour me constituer en centre assimilateur de l'infini: amené à l'évidence d'une limite vide, je suis illimité sans idée de grandeur, sans référence métaphysique. (Barthes, 1971, p. 45)

Or, ce pays sans frontière ouvert à un sujet vide n'en reste pas moins délimité pour mieux être défendu. Par quoi? Des images. Un dispositif d'images. Désir d'images. Et, à considérer la constellation de livres, de textes, de cours, d'entretiens, de thèmes divers dont procède l'œuvre de Barthes, à distinguer les pays dont il a parlé et dans lesquels il a pu voyager - au premier rang desquels la Grèce - à observer la place et la pratique régulière du fragment dans le discours, il est peut-être (paradoxe) une image, l'Image - le Paysage - , qui constitue ce dispositif, pour en régler la complexité de «voyage en sept stations » : l'Archipel. Que recouvre cette Image? Quel rapport entretient-elle avec notre besoin d'images? Et celles-ci avec le dessein (le dessin?) du Vide? C'est en répondant à ces questions que nous espérons comprendre comment la frontière, pour Barthes, vide en soi, est surtout cet espacement, cet intervalle, dans et par lequel s'opère au fond la « réfraction ontologique » :

par quoi l'unité, cette lumière, ayant à nous atteindre à travers des mots aujourd'hui extériorisés, dévie dans leur épaisseur au point que son origine apparaît ailleurs qu'en l'existence, sa substance autre que celle des actes quotidiens, sa forme 
trouble, irrégulière, mouvante (Bonnefoy, 1982, quatrième de couverture).

Notre étude ne porte donc pas directement sur la notion de Frontière au sens commun du terme, géographique ou politique, ou les deux à la fois. Mais on le sait, "[c]omme "la carte est une projection de l'esprit avant d'être une image de la terre (Christian Jacob), la frontière est d'abord une affaire intellectuelle et morale» (Debray, 2013, p.17). C'est cette affaire, ou son paysage mental, qui nous intéresse avant tout dans l'œuvre et dans l'écriture de Barthes. Que ce paysage relève à la fois de l'Archipel et de la Frontière paraît presque secondaire. Presque seulement, car ces images restent en fait liées sinon à des États, du moins à des territoires réels (la Grèce, le Japon, nous venons de le voir). Ainsi, dans notre étude, nous parlerons de la notion de Vide en général, pour mieux situer celle, particulière, de Barthes, en décrire le système dont elle procède : la métaphore de l'Archipel. À la suite de quoi, à l'instar du $M a$ japonais, il sera question du paysage mental du Vide, c'est-à-dire comme pays (de l'écriture), histoire (de ma vie), expérience (de la mort), concept (de Vita Nova), sensibilité (la nuance), avenir (du roman - ou de la lecture) et crainte et nostalgie.

Nous proposons ici de donner seulement un aperçu de chacun de ces thèmes. Et, à chaque fois, de faire le point, de revenir à la notion de frontière.

\section{Le Vide}

Le Vide commence avec le mot lui-même. 
Le Vide, c'est d'abord, au plus haut de sa racine étymologique (indoeuropéenne), le désert. Le Vide, s'opposant au Plein, s'apparente au Néant. Et ce sens n'a au fond guère évolué. Du moins dans son usage classique. En effet, si le Vide correspond au rien, c'est à la mesure de ce que, au carrefour de son propre étymon (res, rei) et du Vide selon la science moderne, il renvoie à quelque chose.

Le Vide, en effet, avec la physique (quantique en particulier), n'est plus conçu comme un état statique par opposition au Plein d'une chose dynamique. Pascal l'a bien dit: « Il y a autant de différences entre le néant et l'espace vide que de l'espace vide au corps matériel; et [...] ainsi l'espace vide tient le milieu entre la matière et le néant.» (Pascal, p. 189) Bien plus, à force de découper, de classer, de combiner et de réinventer la matière du monde, il s'avère que plus la portion prélevée est réduite, plus on se glisse dans l'intervalle, la frontière, qui la sépare des autres portions, et plus celui-ci se voit attribuer une forme qui est aussi une force puissante. Ainsi «le vide est peuplé d'états virtuels qu'une excitation peut révéler: c'est le principe de l'apparition des paires particuleantiparticule. Le vide quantique se comporte comme un milieu polarisable capable d'écranter l'action des objets qu'il côtoie. » (Pire, 2008) Or, une telle conception n'est pas étrangère à Barthes. Parce qu'elle lui permet de soutenir sa propre idée du Vide. Quand, pour le dire vite au détour d'un entretien, il affirme que «le vide, c'est plutôt le nouveau, le retour du nouveau » (Barthes, 2002a, t. III, p. 995) — par opposition au Plein, moins en tant que substance en général que, plus particulièrement, «mauvaise forme: le plein, c'est subjectivement, le souvenir (le passé, le Père), névrotiquement la répétition, le stéréotype »-, il s'appuie sur la théorie du 
bootstrap de Chew et Mandelstam selon laquelle «l'ensemble des particules s'engendrerait lui-même (self-consistance) » pour préciser aussitôt, par métaphore, que «le vide dont nous parlons, ce serait en somme la self-consistance du monde» (p. 996).

Mais Barthes a également su mettre en œuvre cet « écrantage », aménager dans son discours, dans ses textes, le lieu et le dispositif énergétiques, métaphoriques, permettant de lier, de filer des ensembles de langage, des unités de sens pour les faire interagir, les faire se déplacer au profit du signifiant, non du signifié : quelque chose comme « un espace nouveau où le sujet de l'écriture et celui de la lecture n'ont pas exactement la même place» (Barthes, 2002a, t. IV, p. 204). Nous pensons notamment à l'image de la boîte noire de «Par où commencer ». Dans ce texte, où il est question de méthode d'analyse structurale, elle intervient pour lier deux ensembles: un étudiant (disposé à la critique littéraire) et un roman (L'île mystérieuse de Jules Verne), c'est-à-dire la lecture et l'écriture. Or l'intervention n'est pas première mais secondaire. Barthes tient l'image d'un linguiste soviétique, I. I. Revzin. Pour celui-ci, il s'agit d'un motif analogique tiré de la cybernétique illustrant le fait qu'une description scientifique a pour fonction de dire " comment s'effectue le passage de $\mathrm{A}$ à $\mathrm{B}$ et quelles sont les liaisons entre ces deux ensembles» (p. 87). Et quand Barthes, lui, conçoit qu'«il faut en somme définir le passage d'un équilibre à un autre, traverser la "boîte noire" ", l'image, pour autant qu'elle représente une forme du Vide, se fait metaphora, "métaphore littérale» (au sens de transport) dans laquelle s'inscrit tout le travail d'analyse structurale puisqu'elle actualise, déjà, la conclusion du texte: elle offre «à la fois le moyen de commencer l'analyse à partir de quelques codes 
familiers et le droit de quitter ces codes (de les transformer) en avançant, non dans le texte (qui est toujours simultané, volumineux, stéréographique), mais dans son propre travail » (p. 94).

Le Vide, cela dit, est une notion que Barthes emprunte surtout à la pensée extrême-orientale. Plus précisément, il s'agit d'une notion si fondamentale, si structurale pour cette pensée que le statut même de notion convient mal. Principalement issu du taoïsme chinois, le Vide est «à la fois cet état suprême de l'Origine "(le nouménal)" et l'élément central dans le rouage du monde “(le phénoménal)" » (Cheng, 1991, p. 53), et ce qu'il offre "n'est plus tant une "explication" [...] qu'une "compréhension", une "entente", et finalement une sagesse proposant un art de vivre » (p. 49). Autrement dit, si pour les Occidentaux (nous), le Vide s'inscrit plutôt dans un rapport à la matière, l'ExtrêmeOrient le fait participer du mouvement, des mutations infinies qui animent la matière et ses avatars. C'est en fonction de ce principe que l'on conçoit qu'il est «à la fois le chemin à parcourir et la fin du parcours, la méthode et l'accomplissement. [...] À peine est-on engagé dans le chemin, qu'on l'a parcouru tout entier » (Grenier, 1992, p. 14). Et que Barthes, lui, préfère "l'idée de décentrement» (2002a, t. 3, p. 995).

Mais son œuvre en appelle régulièrement au Vide. Non seulement par de nombreuses références au Tao, au Zen, mais encore par l'usage du fragment, de la note. Dans le cours sur le Neutre, la notion correspond d'autant mieux à une autre idée du Vide que, d'une part, Barthes s'appuie sur des traits fondamentaux du Zen et du Tao, comme le satori, le wou-wei : « ne pas diriger, ne pas finaliser sa force, la laisser sur place» 
(Barthes, 2002c, p. 222-223); et que, d'autre part, la préparation du cours, la disposition des fragments participent d'un projet qui vise à déjouer la maîtrise, à faire que le sens ne prenne pas: un projet qui est en même temps «une introduction au vivre, un guide de vie (projet éthique) : je veux vivre selon la nuance» (p. 37). Néanmoins, Barthes rapproche et parfois superpose - identifie? - au Vide extrême et oriental des notions de notre culture occidentale. Le satori zen «brusque débouché sur le vide: [...] sorte de catastrophe mentale qui se produit d'un seul coup» (p. 220) - apparaît dans le cadre du kairos grec - «un élément, un temps énergétique : le moment en soi en tant qu'il produit quelque chose, un changement: c'est une force» (p. 218). Mais nous pensons surtout au début du texte qui ouvre les Essais critiques, consacré à la peinture des maîtres hollandais :

Il y a dans les musées de Hollande un petit peintre qui mériterait peut-être la renommée littéraire de Vermeer de Delft. Saenredam n'a peint ni des visages ni des objets, mais surtout l'intérieur d'églises vides, réduites au velouté beige et inoffensif d'une glace à la noisette. Ces églises, où l'on ne voit que des pans de bois et de chaux, sont dépeuplées sans recours, et cette négation-là va autrement loin que la dévastation des idoles. Jamais le néant n'a été si sûr. (2002a, t. II, p. 283).

C'est comme si Barthes se situait à la croisée, à la frontière, des mondes.

\section{Le pays de l'écriture}

Dans ces conditions, nous concevons facilement que le Japon, qui abrite d'autant mieux la pensée du Vide qu'il semble l'avoir poussée, avec le Zen, dans les retranchements formels extrêmes des modes de vie, soit le pays d'élection de Barthes. D'autant 
plus que, de retour d'un voyage en 1968, il lui a consacré un livre, L'Empire des signes. Reste que, nous l'avons dit, le Japon de Barthes n'est pas simplement le pays réel, auquel s'intéresserait un ethnologue ou un touriste, ni strictement le pays symbolisant d'autres façons de vivre et de penser. En bon artisan de l'écriture, qui sait que son art a pour finalité «l'exposition de ses moyens, dans le suspens évocateur des significations » (Haddad, 2006, p. 842), le Japon constitue en fait l'espace, le dispositif imagé et imageant, dans et par lequel il va loger, étendre et découvrir in fine son art. "Pourquoi le Japon? parce que c'est le pays de l'écriture » (1971, encart dépliant), annonce d'emblée Barthes. Et au début du premier fragment, pour mieux situer le Japon (son Japon) dans le no man's land vide, mais dynamique, de la littérature (référence à Henri Michaux à l'appui), il prend soin de bien délimiter la frontière du rêve et de la réalité, de circonscrire au mieux la zone de réalité profonde où l'écrivain et l'objet de l'écriture semblent indissociables - où, en somme, "le fait de pouvoir dire "je", d'être sujet, c'est d'occuper un site, une position où l'on se met au centre de son monde pour pouvoir le traiter et se traiter soi-même » (Morin, 2005, p. 88) :

Si je veux imaginer un peuple fictif, je puis lui donner un nom inventé, le traiter déclarativement comme un objet romanesque, fonder une nouvelle Garabagne, de façon à ne compromettre aucun pays réel dans ma fantaisie (mais alors c'est cette fantaisie même que je compromets dans les signes de la littérature). Je puis aussi, sans prétendre en rien représenter ou analyser la moindre réalité (ce sont les gestes majeurs du discours occidental), prélever quelque part dans le monde (làbas) un certain nombre de traits (mot graphique et linguistique), et de ces traits former délibérément un système. C'est ce système que j'appellerai : le Japon. (Barthes, 1971, p. 9) 
Ce qui compte ici, c'est «là-bas». Et d'autant mieux qu'il participe, poétiquement parlant, de cet « ailleurs fantasmatique visant non pas tant à découvrir d'autres symboles, une autre métaphysique, qu'à fissurer son propre système symbolique par la fiction d'un Neutre » (Comment, 2003, p. 68). Or cet élan vers un autre monde, cet assaut vers une nouvelle frontière, un autre pays, un autre système le représente, le réalise sinon plus foncièrement que le Japon, du moins originellement : la Grèce.

Des textes parlant de ce pays comme le fait le livre sur le Japon, on ne compte qu'En Grèce. Il s'agit d'un petit texte, un des premiers de Barthes, qui relate le souvenir d'un voyage effectué en 1938 dans l'archipel des Cyclades. En quelques fragments, parfois quelques lignes, il y est question de la vie à Athènes, de musées et statues, de nourriture, de fleurs, de sites antiques et d'îles, du voyage d'île en île. Dès le début, d'une impression générale de Vide, par décentrement : «En Grèce, il y a tant d'îles qu'on ne sait si chacune est le centre ou le bord d'un archipel. C'est aussi le pays des îles voyageuses : on croit retrouver plus loin celle qu'on vient de quitter. » (Barthes, 2002a, t. I, p. 68) Une impression qui non seulement va traverser horizontalement le texte, le paysage de part en part, mais encore va se déployer vers le cœur même des Cyclades et découvrir verticalement, au sommet de l'île de Délos, comment l'espace du dehors va de pair avec «l'espace du dedans » (pour emprunter un titre de Michaux) : "Il faut gravir le Cynthe à midi, à l'heure la plus perpendiculaire, l'heure droite et profonde où la flamme solaire va au cœur des terres, au fond des poitrines, et trace sur les yeux comme un signe, une blessure de feu, sèche comme une blessure d'amour. » (p. 74) 
Or, si la Grèce est l'autre pays du Vide, c'est aussi parce que Barthes trouve en lui le premier paysage de l'écriture en l'espèce de la tragédie, et inversement. N'oublions pas, en effet, ce que dit Barthes de celle-ci, dans «Culture et tragédie», son tout premier texte: elle "n'est qu'un effort ardent pour dépouiller la souffrance de l'homme, la réduire à son essence irréductible, l'appuyer - en la stylisant dans une forme esthétique impeccable - sur le fondement premier du drame humain, présenté dans une nudité que seul l'art peut atteindre » (p. 30).

\section{Histoire de ma vie}

Du séjour au Japon aux vacances en Grèce, la vie de Barthes semble la réserve dans laquelle sa pensée et son écriture trouvent leur inspiration ou, dans la perspective du Vide, leur aspiration... D'autant que la vie et le Vide ne sont peut-être que les deux faces d'une même pièce.

D'un côté, il ne faut pas oublier les circonstances dans lesquelles Barthes commence à écrire : la guerre, la maladie. Ses tout premiers textes sont en effet parus sous l'Occupation, principalement dans la revue Existences des étudiants du sanatorium de Saint-Hilaire-Du-Touvet, où il soigne sa tuberculose. Jusqu'à la Libération, il demeure dans ce village des Alpes, loin de chez lui, de sa mère (son père est mort peu après sa naissance, durant la Première Guerre mondiale). Or, bien que grave (la tuberculose est une maladie mortelle à l'époque), la situation n'est pas exclusivement malheureuse pour Barthes. La vie communautaire est en effet active, pleine. Il assiste à des conférences, lit beaucoup, participe à la refonte de la revue, sur le 
fond et sur la forme, et met en œuvre ce qui constituera sa méthode de travail tout au long de sa vie: la fiche, la note (en particulier avec l'œuvre de Michelet). Parce que, question de mémoire, «il faut être désinvolte dans le travail, savoir saisir l'essentiel et sacrifier le reste» (Calvet, 1990, p. 84), aurait alors conseillé Barthes à un ami. Mais aussi parce que, question de style sur laquelle il revient dans La Préparation du roman, « ce que la Mémoire doit préserver, ce n'est pas la chose, c'est son retour, car ce retour a déjà quelque chose d'une forme - d'une Phrase» (Barthes, 2003, p. 139). Bref, «la tuberculose consacre [...] une coupure décisive, en libérant le temps intense d'un apprentissage» (Roger, 1990, p.402). Pas seulement une instruction technique, pratique, mais une éducation morale et sentimentale puisqu' « au sana, on trouve tous les affects qu'on veut; aucun besoin du dehors. Une fois la structure (le Vivre-Ensemble) lancée, elle dure à la façon d'un homéostat perpétuel » (Barthes, 2002b, p. 71). Or dans cette vie autarcique, pleine, ce qui fascine Barthes, c'est «l'intuition du vide vertigineux de la plénitude de groupe » (p. 70).

D'un autre côté, et à l'autre bout de son œuvre, Barthes suggère, dans La Préparation du roman, l'exploration d'une "Poétique du Vide ». Quelle en est la finalité?

Eh bien la vie, la sensation de la vie, le sentiment d'existence; et nous savons que ce sentiment, pour être pur, intense, glorieux, parfait, il faut qu'un certain vide s'accomplisse dans le sujet; même lorsque la jubilation (d'amour) par exemple est la plus intense, c'est parce qu'il y a dans le sujet un vide de langage lorsque le langage se tait, qu'il n'y a plus de commentaire, d'interprétation, de sens, c'est alors que l'existence est pure: cœur «plein» (qui «déborde») = connaissance d'un certain vide (thème éminemment mystique); 
le défaut, la défaillance de discours renvoie à deux états extrêmes : la misère absolue du "paumé », la jubilation ardente du « vivant». (Barthes, 2003, p. 84)

La poétique du Vide procède donc d'une éthique de vie. $\mathrm{Ou}$, si l'on veut (vous excuserez le mot-valise), d'une sorte de poéthique consistant à séparer, selon un partage relevant de «la misère absolue » et de « la jubilation ardente », la mort et la vie, le Plein et le Vide, ou encore le néant et l'être. Soit une certaine forme de représentation de l'union des extrêmes. Et, ce faisant, de leur frontière, du mouvement qui les sépare, de la force de Résistance entre eux.

Conduire l'écriture sous l'égide de cette force est le pari auquel Barthes semble s'être livré, totalement et infiniment, tout au long de son œuvre. Initialement dans la pensée de Barthes, cette force participe de la tragédie, « car elle n'existe pas à l'état spontané; elle se crée avec de la souffrance et de l'art; elle présuppose de la part du peuple une culture profonde, une communion de style entre la vie et l'art » (Barthes, 2002a, t. I, p.32). Idéalement, si cette communion caractérise le héros tragique, Barthes finit par en identifier le propre chez l'artiste qui, à chaque œuvre, doit

affronter en soi ces spectres de la subjectivité moderne, dès lors qu'on n'est plus prêtre, que sont la lassitude idéologique, la mauvaise conscience sociale, l'attrait et le dégoût de l'art facile, le tremblement de la responsabilité, l'incessant scrupule qui écartèle l'artiste entre la solitude et la grégarité (Barthes, 2002a, t. V, p. 904-905).

Personnellement ou historiquement, cet affrontement interne s'est très vite présenté à Barthes comme le moyen même de la (de $s a$ ) condition, critique et littéraire : 
Je veux dire que je ne puis me prêter à la croyance traditionnelle qui postule un divorce de nature entre l'objectivité du savant et la subjectivité de l'écrivain, comme si l'un était doué d'une «liberté » et l'autre d'une "vocation», propres toutes deux à escamoter ou à sublimer les limites réelles de leur situation: je réclame de vivre pleinement la contradiction de mon temps, qui peut faire d'un sarcasme la condition de la vérité. (Barthes, 2002a, t. I, p. 676)

Enfin, poétiquement, ce style de présence, c'est «par opposition à toute forme de lutte guerrière, comme ce paradoxal pouvoir d'inertie, cette étrange capacité d'immobilité, à quoi l'on reconnaît ceux qui refusent la compromission avec les puissances du temps » (Dastur, 2001, p. 164). Sans quoi, à l'appel que lance Barthes dans un texte consacré à «l'œuvrevie » (2002a, t. V, p. 465) de Proust - « [c]'est l'intime qui veut parler en moi, faire entendre son cri, face à la généralité, à la science » - ne répondrait pas, dans la partie où il découvre et imagine sa propre œuvre-vie, sa «Vita Nova » (p. 467), ceci :

Peut-être est-ce finalement au cœur de cette subjectivité, de cette intimité même dont je vous ai entretenus, peut-être est-ce à la « cime de mon particulier » que je suis scientifique sans le savoir, tourné confusément vers cette Scienza Nuova dont parlait Vico : ne devra-t-elle pas exprimer à la fois la brillance et la souffrance du monde, ce qui, en lui, me séduit et m'indigne? (p. 470)

\section{Expérience de la mort}

Quand Barthes parle des contradictions, des luttes de son temps, on peut aussi comprendre combat contre la marche du temps, contre la mort. L'expérience du sanatorium aura en cela 
été fondamentale. Mais elle aura aussi été fondatrice. La mort en effet, latente alors dans la réalité, n'est pas considérée comme la fin annoncée de la vie. Au contraire, elle est conçue, sinon comme la substance même de la vie (ou une nouvelle forme de vie qu'il faut apprivoiser), du moins comme un autre état de la matière que l'on peut modeler. D'emblée, dans "Culture et tragédie », Barthes dit non au Temps et oui à l'Instant, à l'ici et maintenant du monde comme il va :

Peu importe de savoir comment finira le monde, ce qu'il importe de savoir, c'est ce qu'il est, quel est son véritable sens - non point dans le Temps, puissance fort contestable et contestée, mais dans un univers immédiat, dépouillé des portes mêmes du Temps. (2002a, t. I, p. 31)

En fait, Barthes conçoit la mort de deux façons. Il y a un bon et un mauvais temps. Le bon, c'est celui qui est ouvert et consacré par la «mère » Histoire (via Michelet), par l'écriture du temps qui passe dans la mesure où elle est conforme à la nature, au corps :

la fin de l'histoire, c'est de retrouver dans chaque chair du passé l'élément corruptible par excellence, non pas le squelette mais le tissu [...], allons au plus fragile, laissons là l'expression, les traits, et retrouvons la substance corruptible et mortelle, la couleur du sang, la densité des tissus, le grain de la peau, tout ce qui s'effondrera et s'écoulera au cercueil (Barthes, 1974, p. 78).

Le mauvais temps, c'est celui qui, inversement, relève de l'histoire de la Mère. Je veux dire que l'Histoire se personnifie avec la figure maternelle. Notamment avec la Mère Courage de Brecht, à laquelle Barthes consacre plusieurs textes. Par cette personnification, l'écriture du temps, de type théâtral et engagé, ne participe plus de la vie la plus charnelle, mais la plus intellectuelle et active. D'abord, parce que 
Brecht dépayse la fatalité de la guerre, il la fixe en Mère Courage, [...] et par cette opération expérimentale, nous sommes débarrassés de ce que nous pensions être condition poisseuse, fatale, pathétique: la distance du regard nous prépare la liberté du jugement et le pouvoir de l'action (Barthes, 2002a, t. I, p. 505).

Ensuite, parce que, dans cette œuvre de Brecht, Barthes voit comme l'histoire pure, voire l'essence du récit, qui fait de la Mère un personnage fondamental, mythique. À la frontière de la vie et de la mort, avec celle du fils, le statut originel (indifférencié et virtuel) de ce qui est alors la Mère-enfant garantit la force de son rôle fonctionnel (intellectuel et actif); et inversement, ce rôle régissant tout des choses du monde témoigne de la réalité, voire de la nécessité, de la mort :

c'est le fils qui accouche spirituellement de la Mère [...], c'est la Mère qui le reprend, le continue, comme si c'était elle la jeune pousse, la nouvelle feuille appelée à s'épanouir [...], une distance sépare la mère du fils, nous rappelant que cet itinéraire juste est un itinéraire atroce : l'amour n'est pas ici effusion, il est cette force qui transforme le fait en conscience, puis en action: c'est l'amour qui ouvre les yeux (Barthes, 2002a, t. II, p. 402).

Enfin parce que cette expérience extrême, Barthes l'aura vécue à sa manière, littéralement, poétiquement, avec la mort de sa mère, dans les soins qu'il lui aura prodigués: «elle était devenue ma petite fille, rejoignant pour moi l'enfant essentielle qu'elle était sur sa première photo » (Barthes, 1980, p. 112).

Le bon temps relève donc de l'espace de l'Instant, constitué par les profondeurs de la sensibilité. C'est l'« Instant absolument frais : comme si on mangeait la chose notée, sur l'arbre même, comme un animal qui broute l'herbe vive de la sensation» (Barthes, 2003, p.85-86). Or il n'est pas sans 
rapport avec les profondeurs du temps. Il relève d'une conscience temporelle spécifique, à la mesure du futur antérieur, ou plutôt d'un antérieur futur. Suspendu dans des formulations étranges, $c^{\prime}$ est l'« Instant qui a vocation de Trésor : "Demain, le souvenir" [...] : la "mémoire immédiate" comme si la Notatio (le fait de noter) permettait de se souvenir sur-lechamp» (p. 86). Il y a là un paradoxe qui s'explique facilement dans la mesure où la fraîcheur de l'Instant participe de l'écriture, de la Note : « Je crois que c'est un peu la fonction de la Poésie », ajoute Barthes.

Cette instantanéité fonde une «dialectique fine du temps» (p. 85) qui permet de le remonter et de prendre soin des morts, de les rendre à eux-mêmes. L'historien est en effet

celui qui a renversé le Temps, qui revient en arrière, à la place des morts et recommence leur vie dans un sens clair et utile; il est le démiurge qui lie ce qui était épars, discontinu, incompréhensible : il tisse ensemble les fils de toutes les vies, il noue la grande fraternité des morts (Barthes, 1974, p. 75).

L'Instant fonde ainsi une autre logique temporelle, du bouleversement, de la rupture, de la dérive (comme le futur antérieur). Il fonde une poétique de la scission qui unit les extrêmes. Et c'est cette nouvelle logique que Barthes découvre aussi avec la mort de sa mère et qu'il expérimente avec son Journal de deuil : " Dès qu'un être est mort, construction affolée de l'avenir (changements de meubles, etc.) : aveniromanie.» (Barthes, 2009, p. 16)

\section{Concept de Vita Nova}

«Deuil : non pas écrasement, blocage (ce qui supposerait un "rempli"), mais une disponibilité douloureuse : je suis en alerte, 
attendant, épiant la venue d'un "sens de vie" » (p. 90). Dans le Journal de deuil, la narration se veut transparente. Il s'agit de partager une expérience : la mort d'un être aimé, le chagrin de qui lui survit. Mais pas simplement sous une forme directe, pathétique, qui exprimerait la souffrance. C'est sous une forme indirecte, la plus neutre possible, que Barthes énonce d'autant mieux la douleur qu'elle reste sur la corde raide d'une mise en mots et en images dépouillée, mais mesurée, dans le questionnement et le recueillement solitaire de l'auteur et du lecteur. Et quand ce style demande si non seulement il est encore possible de vivre, mais d'écrire, quand les liens qui nous attachent au corps et à l'âme se défont, quand le désir est suspendu et semble disparaître et quand, par contrecoup, on s'identifie à la rupture, au Vide même, il répond en même temps : «Oui! » Or la Vita Nova est le concept dans lequel va se cristalliser ce style minimal. Ou plutôt s'étoffer, se déployer, car l'un des problèmes majeurs relève de la prise en compte d'autres matériaux que ceux de la culture écrite.

Qu'est-ce que la Vita Nova? C'est « l'idée d'une Rupture de Vie, d'une Novation du Genre de Vie, de l'Organisation d'une Nouvelle Vie » (Barthes, 2003, p. 280) qui va investir toutes les pratiques culturelles du sujet, des plus communes aux plus intimes : c'est un changement d'identité. La Vita Nova renvoie à la question: «Mais, qui suis-je?» Dans la lignée de Rimbaud, «[r]ompre veut dire: je vais produire radicalement, sans concession, au prix d'un arrachement total, un autre je» (p. 281). Et d'une façon telle que ce je, omniprésent dans la relation au monde et à soi, dans la contemplation et la réflexion, dans le regard porté sur l'écriture et le désir qui la porte «Vita Nova voulue, fantasmée par l'GEuvre à Faire» (p. 284) — que ce je devienne, en son altérité, un miroir d'encre : 
Le problème pour nous c'est l'œuvre à faire, opus agendum, donc l'œuvre que je dois faire; c'est donc, par un artifice volontaire et provisoire, soi-même qu'il faut sacraliser; il faut faire de soi-même un être sacré, en tant qu'il fait (et non en tant qu'il est), en sorte que les atteintes du monde à l'égard de l'ouvrier (operator) que je suis soient graves. (p. 295)

Cette énonciation en quête d'une écriture de soi se présente sans fard, en constante évolution, mettant en garde le lecteur contre la duplicité de sa voix et contre la difficulté de son projet, car l'inclusion d'une considération d'ordre personnel et religieux marque une rupture avec la pratique littéraire passée et dresse de fines frontières au cœur du mode de vie - dans "le détail de ses goûts, de ses habitudes, de ses régimes» (p. 297) :

La Vita Nova, c'est-à-dire l'entrée dans la Fabrique de l'œEuvre, implique en effet une éducation, une auto-éducation, et donc une rééducation, puisqu'il s'agit de passer d'un genre de vie à une autre. Il s'agit de concevoir subtilement sa vie (son genre de vie), sans crainte d'un sur-moi qui condamnerait cette subtilité comme futilité; d'où la notion de casuistique: force qui ose les distingos subtils dans l'ordre du genre de vie.

\section{Sensibilité : la Nuance}

Tout cela nous ramène directement à la figure noble de la Frontière et du Vide : la Nuance. Elle a pour paysage le nuage. Et avec lui, celui qui écrit se manifeste, se signale dans la conscience intime la plus large et la plus fine de sa pratique, comme de son désir, d'écriture. La Nuance participe d'une «logique rêveuse» (Richard, 2006, p. 48) qui se développe en trois temps :

dans un premier temps elle est mue par un instinct de révolte [...], dans un deuxième temps elle creuse un écart, elle crée la 
possibilité d'une béance [...], la nuance devient en un troisième moment, à partir de son nouvel habitacle infime, une puissance de rayonnement et d'expansivité (p. 48-49).

Dans sa lecture, Richard s'appuie sur La Préparation du roman. Mais elle s'applique aussi à ce que Barthes dit de l'intellectuel dans Mythologies, ou plutôt à l'image que l'homme politique Pierre Poujade s'en fait, dont Barthes analyse et critique (mais pas seulement) le discours :

Comme tout être mythique, l'intellectuel participe d'un thème général, d'une substance : l'air, c'est-à-dire (bien que ce soit là une identité peu scientifique) le vide. [...] Les intellectuels ne sont ni des idéalistes, ni des réalistes, ce sont des êtres embrumés, "abrutis". Leur attitude exacte est celle de la nuée [...]. Suspendus dans le vide supérieur, les intellectuels en sont tout emplis, ils sont "le tambour qui résonne avec du vent" : on voit ici apparaître le fondement inévitable de tout antiintellectualisme : la suspicion du langage, la réduction de toute parole adverse à un bruit, conformément au procédé constant des polémiques petites-bourgeoises, qui consiste à démasquer chez autrui une infirmité complémentaire à celle que l'on ne voit pas, à charger l'adversaire des effets de ses propres fautes, à appeler obscurité son propre aveuglement et dérèglement verbal sa propre surdité. (Barthes, 2002a, p. 814)

La critique de Barthes est sans appel. Pourtant, au cœur de celle-ci, cet air, ce vide, cette nuée, ne dressent-ils pas en quelques traits le pays de frontière de l'intellectuel, la Nuance, " en tant qu'il fait "sous la plume de Barthes" (et non en tant qu'il est "dans le discours de Poujade") »? Nous voyons alors bien comment " opérateur de distinction, elle est en elle-même un opérateur de simultanéité » (Barthes, 2003, p. 83).

Du coup, bien avant d'être énoncée et problématisée dans l'ailleurs et toujours de la Vita Nova, celle-ci n'est-elle pas déjà à l'œuvre, peut-être dès le premier texte? Et systématiquement 
comme «rhétorique du silence» (Genette, 2000, p. 203) : de toutes les choses dont elle peut parler, «elle leur laisse, ou plutôt leur restitue, par une technique très subtile (et qui reste à étudier) d'évasion sémantique, ce sens tremblé, ambigu, indéfini, qui est leur vérité » (p. 203-204)?

\section{Le roman (ou la lecture) de l'avenir}

Un jour, à l'université, un professeur s'étonnait du virage que prenaient les études de l'œuvre de Barthes parce qu'elles la traitaient comme une œuvre romanesque. Et, de la première partie du Métier d'écrire d'Éric Marty - un récit dans lequel il parle de sa rencontre avec Barthes et de leurs entrevues amicales à Paris - à la fiction de Jean Esponde, Roland Barthes, un été (Urt 1978) - qui imagine la vie, le vide de Barthes après la mort de sa mère dans sa maison de vacances - , c'est l'écriture romanesque actuelle qui s'intéresse plus ou moins directement à cette œuvre. Si Barthes s'est, durant deux années au Collège de France, intéressé en profondeur à La Préparation du roman, donnant ainsi suite au désir de roman qu'il a eu l'occasion d'exprimer à plusieurs reprises, l'avenir du roman de Barthes n'est-il pas aujourd'hui en nous, ses lecteurs?

Cette vision paraîtra quelque peu futile. Néanmoins, nous parlons sous la caution de Barthes dans la mesure où, consciemment ou non, il semble avoir anticipé cette situation. N'oublions pas ce qu'il nous dit: «je ne suis pas là où vous m'attendez ». Et ailleurs : « je ne parle pas là où ça est, je parle à côté » (Barthes, 2003, p. 392). Or, à côté, qu'il y a-t-il? Le lecteur, justement. Barthes a toujours eu cette conscience que, pour connaître l'écriture, il faut aussi connaître, reconnaître, la 
lecture. C'est ce que la fin de la célèbre "Mort de l'auteur » avance : "nous savons que, pour rendre à l'écriture son avenir, il faut renverser le mythe : la naissance du lecteur doit se payer de la mort de l'Auteur » (Barthes, 2002a, t. III, p. 45). Mais l'idée apparaît dès son séjour en sanatorium, au terme d'un compte rendu polémique d'un numéro de la revue Confluences consacrée au roman :

comme c'est l'amour qui a guéri Lucile la futée et ses sœurs muettes, je crains que le roman ne parle à nouveau que devant ceux qui l'aiment sans trop disserter, c'est-à-dire d'abord ceux qui l'écrivent, et ensuite ceux qui le lisent, s'y perdent et s'y enchantent jusqu'à la pure sagesse de l'aimer en silence (Barthes , 2002a, t. I, p. 53).

L'attaque est dure, et l'on a pu dire que la formule, "poussant très loin le mépris du "métalangage", équivaut à une mise au chômage de la critique » (Roger, 1990, p. 407). Mais peut-être n'a-t-on pas encore assez vu combien la lecture est la clef du roman pour Barthes? En ceci qu'elle est une mise en chantier du romanesque, une mise au travail du désir d'écrire. Parce que « la lecture, c'est précisément cette énergie, cette action qui va saisir dans ce texte, dans ce livre, cela même "qui ne se laisse pas épuiser par les catégories de la Poétique" » (Barthes, 2002a, t. IV, p. 936), lit-on à la fin de «Sur la lecture »; de même que le roman dont il explore la préparation est cet «objet fantasmatique qui ne veut pas être pris en charge par un métalangage» (Barthes, 2003, p.37) et qui «part de quelques romans; en cela prend appui (départ) sur quelque chose qui est comme le Premier Plaisir (de lecture)» (p.39) - et que la lecture est «l'hémorragie permanente, par où la structure [...] s'écroulerait, s'ouvrirait, se perdrait, conforme en cela à tout 
système logique qu'en définitive rien ne peut fermer » (Barthes, 2002a, t. IV, p. 936).

Bref, le roman de Barthes est un livre en puissance, systématiquement renvoyé, ou projeté, dans la lecture en tant qu'elle participe, tel le $M a$ japonais, de cette Frontière : «toute relation, toute séparation entre deux instants, deux lieux, deux états » (Barthes, 2002a, t. V, p. 475). En tant qu'elle constitue un jeu d'ombre et de lumière de l'écriture, du désir d'écrire : face claire, le plaisir du texte, du roman écrit, qui «peut se définir par une pratique (sans aucun risque de répression) : lieu et temps de lecture: maison, province, repas proche, lampe, famille là où il faut, c'est-à-dire au loin et non loin » (Barthes, $2002 \mathrm{a}$, t. IV, p. 251); face sombre, les textes de jouissance qui suscitent, excitent le désir d'écrire un roman :

le plaisir en pièces; la langue en pièces; la culture en pièces. Ils sont pervers en ceci qu'ils sont hors de toute finalité imaginable [...]. Le texte de jouissance est absolument intransitif. Cependant, [...] c'est l'extrême de la perversion qui la définit : extrême toujours déplacé, extrême vide, mobile, imprévisible.

Et en tant que ce jeu est joué et créé par un seul homme, ou une intelligence unique dont la lecture contient et déploie le cœur. Ce qui manquait à la revue Confluences: «On y sent trop l'auteur, pas assez l'homme » (Barthes, 2002a, t. I, p. 53) Ce que ne rate pas la conférence sur Proust, au moment où Barthes en vient à parler de lui, de son désir de roman, de son Moi :

« Moi » doit s'entendre ici lourdement: ce n'est pas le substitut aseptisé d'un lecteur général (toute substitution est une asepsie); ce n'est personne d'autre que celui à qui nul ne peut se substituer, pour le meilleur et pour le pire. C'est l'intime qui veut parler en moi, faire entendre son cri, face à la généralité, à la science. (Barthes, 2002a, t. V, p. 465) 


\section{Crainte et nostalgie}

Ce cœur intelligent demeure partagé, cela dit. Une des émotions que procure à Barthes la lecture, dans la conférence sur Proust où il expose son désir de roman, «c'est que ce sentiment qui doit animer l'œuvre est du côté de l'amour», en ce qu'il est relié au chef-d'œuvre du passé, « proche, transparent, fraternel, bien que nous en soyons éloignés par toute la distance du génie » (Barthes, 2002a, t. I, p.53). Dans cette exposition, le Roman recouvre des missions très modernes. Par rapport aux êtres aimés, il s'agit de "témoigner qu'ils n'ont pas vécu (et bien souvent souffert) "pour rien” » (Barthes, 2002a, t. V, p. 469), de «permettre la représentation d'un ordre affectif, pleinement, mais indirectement» et de créer une forme qui «ne fait pas pression sur l'autre (le lecteur); son instance est la vérité des affects, non celle des idées ». Reste que cette modernité est empreinte de valeurs anciennes, de style classique : «quoi? La bonté? La générosité? La charité? Peut-être tout simplement parce que Rousseau lui a donné la dignité d'un "philosophème" : la pitié (ou la compassion)» (p.468). Et ce sont elles qui réalisent à la fois la limite et le passage entre l'ancien et le moderne, l'essai et le roman.

Mais ces valeurs sont aussi menacées. Quand Barthes s'interroge sur ce changement de vie dans La Préparation $d u$ roman, il invoque le «sentiment qu'il faut se défendre, que c'est une question de survie » (2003, p. 30). Se défendre contre quoi? Contre la petite-bourgeoisie. Contre les nouveaux médias où elle règne. Contre le fait que « les mots deviennent des mythes inconscients, ils entrent au service de ce pouvoir mou (presque anonyme) que détiennent aujourd'hui la presse, la radio, la télévision: on nous parle de plus en plus, et nous parlons de 
moins en moins bien » (Barthes, 2002a, t. V, p. 925). Et que, du coup, l'avenir de la phrase, garante de la littérature, est sinon compromis, du moins fait de compromissions. D'où la question d'une analyse fine de l'esthétique de ces médias, afin de savoir quelles valeurs ils véhiculent en silence. Aussi, la seconde émotion de Barthes est-elle une peur liée au temps qui passe : «quelles sont les forces réelles que mon âge implique et veut mobiliser?» (p.466) Trois éléments sont ici en jeu: le sentiment de la mort, le sentiment de la répétition du travail, de l'écriture, et le fait qu'un événement, le deuil en particulier, "peut survenir, qui va marquer, inciser, articuler cet ensablement progressif du travail, et déterminer cette mutation » qu'est la Vita Nova.

Le plus étonnant, dans ce jeu intellectuel et émotif d'exposition du désir de roman, c'est que Barthes se soumet luimême au principe du Vide qu'il illustre : la simulation. Parce que du roman, si nous savons tout de sa préparation, nous ne voyons et ne verrons rien de sa production. L'écriture s'est seulement déplacée de la sensibilité de l'intellectuel vers celle, plus fine, plus simple et plus calme, de l'Amateur : "Je fais une hypothèse et j'explore, je découvre la richesse de ce qui en découle; je postule un roman à faire, et de la sorte je peux espérer en apprendre plus sur le roman qu'en le considérant seulement comme un objet déjà fait par les autres » (p. 470). Ici, la pensée se lie, s'écrante, à une connexion logique dont nous avons déjà parlé: l'hypothèse, le "Comme si », formule qui relève d'ailleurs moins de la « connexion logique » (occurrence de linguiste) que de ces mots de liaison (expression d'écolier) qui ne signifient rien, sinon la constitution de la Frontière, du Pont entre critique et romanesque, passé et avenir, auteur et lecteur : 
[S]i j'étais écrivain, et mort, comme j'aimerais que ma vie se réduisît, par les soins d'un biographe amical, à quelques détails, à quelques goûts, à quelques inflexions, disons: des "biographèmes", dont la distinction et la mobilité pourraient voyager hors de tout destin et venir toucher, à la façon des atomes épicuriens, quelque corps futur, promis à la même dispersion. (Barthes, 2002a, t. III, p. 705-706) 


\section{Bibliographie}

BARTHES, Roland. (1971), L'Empire des signes, Genève, Skira, coll. « Les sentiers de la création».

—. (1974 [1954]), Michelet, Paris, Seuil, coll. «Écrivains de toujours $"$.

—. (2002a [1993-1994-1995]), CEuvres complètes, Éric Marty (éd.), Paris, Seuil, 5 vol.

-. (2002b), Comment vivre ensemble. Simulations romanesques de quelques espaces quotidiens, cours et séminaires au Collège de France (1976-1977), établi, annoté et présenté par Claude Coste, É. Marty (éd.), Paris, Seuil / IMEC, coll. « Traces écrites».

—. (2002c), Le Neutre, cours au Collège de France (1977-1978), établi, annoté et présenté par Thomas Clerc, Éric Marty (éd.), Paris, Seuil / IMEC, coll. « Traces écrites ».

-. (2003), La Préparation du roman, vol. I et II, cours et séminaires au Collège de France (1978-1979 et 1979-1980), établi, annoté et présenté par Nathalie Léger, É. Marty (éd.), Paris, Seuil / IMEC, coll. « Traces écrites ».

- (2005 [1980]), La Chambre claire. Note sur la photographie, Paris, Gallimard / Seuil, coll. « Cahiers du cinéma ».

—. (2009), Journal de deuil, Paris, Seuil.

Bonnefoy, Yves. (1982 [1972]), L'Arrière-pays, Paris, Flammarion / Skira, coll. « Les sentiers de la création ».

Calvet, Louis-Jean. (1990), Roland Barthes, Paris, Flammarion. 
CHENG, François. (1991), Vide et plein. Le langage pictural chinois, Paris, Seuil, coll. «Points essais ».

Comment, Bernard. (2003), Roland Barthes, vers le neutre, Paris, Bourgois.

DASTUR, Françoise. (2001), dans Paul Audi et al., Écrire, résister, La Versanne, Encre marine.

DEBRAY, Régis. (2013 [2010]), Éloge des frontières, Paris, Gallimard, coll. « Folio ».

Genette, Gérard. (2000 [1966]), Figures I, Paris, Seuil, coll. « Points. Essais ».

Grenier, Jean. (1992 [1973]), L'Esprit $d u$ Tao, Paris, Flammarion, coll. « Champs ».

HADDAD, Hubert. (2006), Le Nouveau Magasin d'écriture, Paris, Zulma.

MORIN, Edgar. (2005 [1990]), Introduction à la pensée complexe, Paris, Seuil, coll. «Points. Essais ».

PASCAL, Blaise. (1858), « Réponse de Pascal au Père Noël », dans OEuvres complètes, t. II, Paris, Hachette, p. 189.

PIRE, Bernard. (2008), "Vide quantique», dans Encylopædia Universalis, Cdrom version 13, Encylopædia Universalis France.

RICHARD, Jean-Pierre. (2006), Roland Barthes, dernier paysage, Lagrasse, Verdier.

RoGER, Philippe. (1990 [1986]), Roland Barthes, roman, Paris, LGF, coll. « Le Livre de Poche Biblio essais ». 


\section{Résumé}

Dans sa recherche d'une langue qui accueille l'autre, ou l'ailleurs, le Vide est pour Roland Barthes une notion fondatrice et limite. $\mathrm{Si}$, pour des raisons culturelles, il la tire essentiellement du Japon, et originellement de la Grèce, un tour d'horizon dans son œuvre montre qu'elle relève en fait, structurellement - géographiquement et littérairement, via la géométrie et le goût du fragment -, d'un paysage en forme d'Archipel, d'un voyage sans attache fixe (apparemment). C'est cette Dérive d'île en île, de fragment en fragment - la délimitation précise donnant, comme l'insularité dont parle Régis Debray dans son Éloge des frontières, «un fond d'homogénéité » - , qui fait de la Frontière, en contrepoint, un thème plus important qu'il ne semble être dans l'œuvre de Barthes. Peut-être négativement, sous l'espèce du sansfrontière? Non. Bien au contraire, car le Vide apparaît comme une force permettant de "soutenir la cause décriée des lisières et des confins », pour reprendre Debray. De sorte que s'il y a une poétique du Vide dans l'œuvre de Barthes, il y a aussi une poétique de la Frontière et que, pour amorcer une première, et rapide, description de cette poétique réversible, nous avons cru bon de nous porter nous-mêmes à la limite de la notion de Vide - donc au plus près du thème de la Frontière - en inscrivant la démarche de notre étude dans les pas d'un petit texte de Barthes consacré à la notion de $M a$ dans l'architecture japonaise. Il est donc ici question du Vide - de la Frontière comme pays (de l'écriture), comme histoire (de ma vie), comme expérience (de la mort), comme concept (de Vita Nova), comme sensibilité (la Nuance), comme avenir (du roman — ou de la lecture) et comme crainte et nostalgie. 


\begin{abstract}
In his search for a language that welcomes the "someone else", or "somewhere else", the Void is, for Roland Barthes, a founding and borderline notion. He draws it mainly from Japan, for cultural reasons, and originally from Greece, but a brief overview of his work shows that it structurally actually relates to an archipelago-shaped landscape or a journey with no ties (geographically, literarily, through geometry and a liking for the fragment). In contrast, this Drift from island to island, from fragment to fragment made the Border a more important theme than it seems in Barthes' work (the precise demarcation conveys "some homogeneity", as the insularity which Régis Debray mentions in his Eulogy of the Frontiers). In a negative way, perhaps, as a kind of "borderless"? No. Quite the opposite, as discreet as it may be (probably because of this discretion), and in relation to the Void, the Border is the positive force aiming to "support the criticized cause of edges and fringes", if we quote Debray. So that if there is a poetics of the Void in Barthes' work, there is also a poetics of the Border. In order to begin a first and quick description of this reversible poetics, we saw fit to make our way to the edge of the notion of Void (hence as closely as possible to the theme of the Border?). We did so by setting the organization of our study within the frame of a small text by Barthes that is dedicated to the notion of $M a$ in Japanese architecture. So here the Void is being considered (and the Border) as country (of writing), as story (of my life), as experience (of death), as concept (of Vita Nova), as sensitivity (the Hue), as future (of the novel-or of reading) and as fear and nostalgia.
\end{abstract}

